\title{
A limited form of granulomatosis with polyangiitis in an ulcerative colitis patient receiving sulfasalazine
}

Fahdah Alokaily, FRCP, ABIM, Reem AlKathiri, MBBS, Sarah AlKathiri, Medical student, Seham Alrashidi, MD.

\begin{abstract}

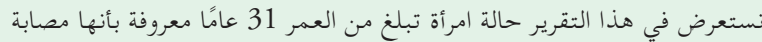

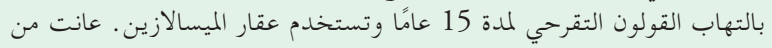

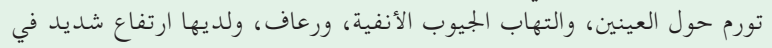

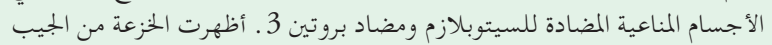

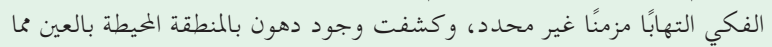

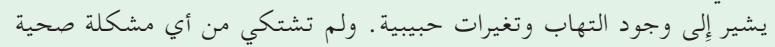

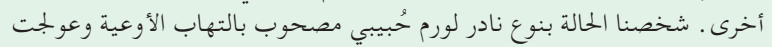

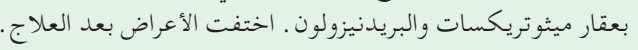

This is a case report of a 31-year-old lady who is known to have ulcerative colitis for 15 years and was on mesalazine. She presented with periorbital swelling, sinusitis, epistaxis, and was found to have positive antineutrophil cytoplasmic antibody and anti-proteinase- 3 of a high titer. Biopsy from the maxillary sinus showed chronic non-specific inflammation and biopsy from the periorbital fat revealed inflammation and granulomatous changes. She had no other organ involvement. She was diagnosed with a limited form of granumatosis and polyangiitis and treated with methotrexate and prednisolone. The symptoms disappeared after treatment.

Keywords: GPA, ulcerative colities, sulfalazine, vasculities

Saudi Med J 2022; Vol. 43 (1): 105-107

doi: 10.15537/smj.2022.43.1.20210914

From the Division of Rheumatology (Alokaily, Alrashidi), Department of Medicine; from the Department of Psychiatry (AlKathiri R), Prince Sultan Military Medical City, from the College of Medicine (AlKathiri S), King Saud University, Riyadh, Kingdom of Saudi Arabia.

Received 20th December 2021. Accepted 30th December 2021.

Address correspondence and reprint request to: Dr. Fahdah Alokaily, Division of Rheumatology, Department of Medicine, Prince Sultan Military Medical City, Riyadh, Kingdom of Saudi Arabia. E-mail:alokaily@yahoo.com

ORCID ID: https://orcid.org/0003-0878-0200
Tnflammatory bowel disease is associated with a 1 variety of extra intestinal manifestation. Ocular involvement have been reported in $2-10 \%$ of patients, and this included: blurred vision, burning or itchy eyes, ocular pain or photophobia, conjunctival or scleral hyperemia, loss of visual acuity, and possible blindness. ${ }^{1}$ Granulomatosis with polyangiitis (GPA) presenting as orbital pseudo tumor was described in a few case reports. ${ }^{2}$ A limited form of GPA (Wegener granulomatosis) has been reported in patients with ulcerative colitis (UC) in the form of pulmonary infiltrate and positive anti-neutrophil cytoplasmic antibody (C-ANCA). ${ }^{3}$ The aim of this case is to highlight the association of an uncommon variety of GPA limited form in a UC patient who is receiving sulfasalazine and the cause effect relationship.

Case Report. A 31-year-old lady was diagnosed with UC 15 years ago when she presented with recurrent hemorrhagic diarrhea and her colonoscopy and colonoscopy biposy confirmed the diagnosis. She was on mesalazine $500 \mathrm{mg}$ twice daily and was in remission for more than 5 years. In addition, she was known to have chronic sinusitis. She complained of swelling of the right eye for 6 months with diplopia but without visual disturbances or redness of eyes. She has a history of nasal discharge and epistaxis without nasal obstruction. She has no fever or headache, no weight loss, and no respiratory symptoms.

Clinical examination revealed proptosis of the right eye with normal movement and vision and the systemic examination was normal. Laboratory investigations showed normal full blood count, eosinophils, erythrocyte sedimentation rate (ESR), and anit-nuclear antibody (ANA) titer was $1 / 80$. Antineutrophil cytoplasmic (C-ANCA) was positive, anti-proteinase-3 (PR-3) was positive with high titer, renal function tests and liver function test were normal, and urine for red blood cell's morphology was normal. Nerve conduction study and chest x-ray were normal. The CT scan orbit showed evidence of orbital swelling with inflammatory 
process (Figures 1\&2). Biopsy from orbital fat showed chronic inflammation with granulomatous changes and based on all of the above, she was diagnosed with a limited GPA.

The patient was treated with prednisolone $60 \mathrm{mg}$ daily (tapered gradually), and methotrexate (MTX) $10 \mathrm{mg}$ weekly. She improved markedly and her symptoms resolved 2 months after treatment. She stopped MTX after one year of remission as she was planning to conceive, and 6 months later, she presented with bilateral orbital swelling. Prednisolone was resumed in addition to azathioprine $100 \mathrm{mg}$ daily, and she achieved remission after 2 months. The C-ANCA then became negative. She was reluctant to receive any drug that can be teratogenic, and this has affected the choice of treatment.

She remained in remission for more than one year afterwards.

Discussion. Extra intestinal manifestations of UC occurs in approximately $25 \%$ of patients. There is an overlap between these manifestations and the complications of UC treatment. Sulfasalazine (SSZ) is widely used in the treatment of UC and it is known to cause many side effects, including a hypersensitivity reaction that resembles pulmonary eosinophilia, bronchiolitis, and interstitial lung infiltrates. ${ }^{4}$ Orbital pseudotumor has not been shown to be a side effect of this medication. Co-existence of SSZ treatment's complications with other clinical conditions were recognized.

Miura et $\mathrm{al}^{5}$ reported a case of necrotizing glomerulonephritis with positive C-ANCA, PR-3, and eosinophilia after resuming treatment with sulfasalazine in a patient with UC. The question of SSZ causing ANCA associated vasculitis (AAV) is to be answered. Adverse events due to SSZ encompass n-acetyltransferase-2 slow acetylator phenotype, long duration of treatment, and high cumulative doses. Several studies have shown that SSZ promotes apoptosis and selectively blocks $\mathrm{NF}^{\mathrm{K}} \mathrm{B}$-dependent gene transcription in various cell types, including neutrophil apoptosis. ${ }^{6}$ The so called neutrophil priming leads to an auto antibody response and production of ANCA. ${ }^{6}$

Disclosure. Author have no conflict of interests, and the work was not supported or funded by any drug company. Dr. Fahdah Alokaily is a member of the Editorial Team, and was therefore excluded from any final editorial decisions regarding this paper.

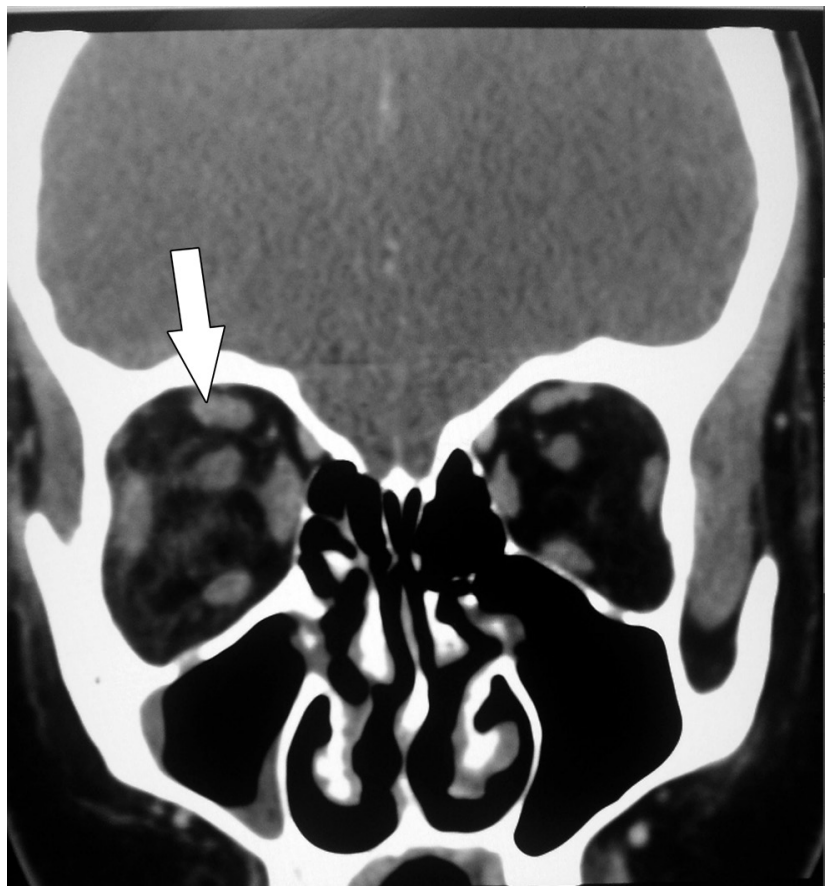

Figure 1 - A computerized tomography scan orbit coronal cut showing bulky right extra ocular muscles with enlarged optic nerve compared with his the left side (inflammatory process).

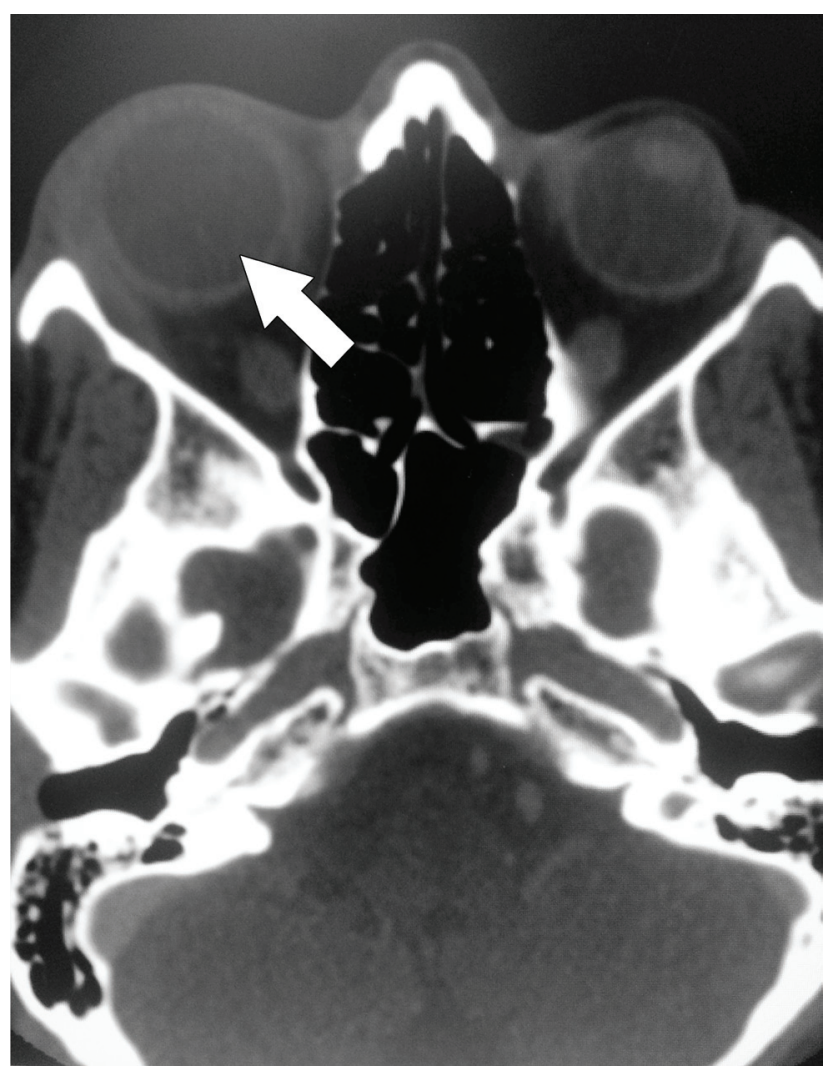

Figure 2 - Axial cut showing right exophthalmos. 
In our case, the co-existence of GPA was supported by the presence of orbital pseudotumor, chronic sinusitis, epistaxis, and positive C-ANCA. The patient improved and achieved remission while on mesalazine, which made it unlikely to be the cause. The pathogenesis of UC was not clear, but it has been emphasized that it is due to immune system dysregulation. The association of UC and GPA with the presence of C-ANCA may indicate a common immunological background. Positive ANCA is present in $40-80 \%$ of UC patients, while (PR-3) ANCA is found to be positive in $6 \%$ of UC patients by ELISA. ${ }^{6}$

Previous case reports regarding the association of a limited form of GPA and UC were mainly in the form of pulmonary involvement in GPA. Yano et $\mathrm{al}^{7}$ reported a patient with bronchiolitis obliterans organizing pneumonitis-like variant in a UC patient. His P-ANCA and drug lymphocyte stimulation test (DLST) for mesalazine were positive and transbronchial biopsy was consistent with GPA. This was labelled as a limited form of GPA in a UC patient. Fayaz et $\mathrm{al}^{4}$ reported a case of a similar scenario with P-ANCA positive and negative C-ANCA and thoracoscopic lung biopsy was consistent with the eosinophilic variant of GPA. The DLST was not performed in this case like most similar reports. Kasuga et $\mathrm{al}^{3}$ described a young lady with UC who had multiple nodular infiltrates in the lungs and increased PR-3 ANCA. She was on mesalazine, which is known to cause pulmonary toxicity. She improved without treatment or discontinuation of mesalazine. The reported cases on the association of UC and GPA, the UC diagnosis preceded the occurrence of GPA by several years, and the GPA presentations were usually in the form of pulmonary involvement. Isse et al, ${ }^{2}$ reported an elderly patient who was known to have GPA and was in remission then she developed exophthalmus and her radiological and histopathological investigation revealed orbital inflammatory pseudotumor which was treated with prednisolone.

Limited form of GPA that is not life threatning can be treated with MTX, rituximab, mycophenolate mofetil, leflunomide, and cyclosporine. Modifying treatment according to the severity of the disease often proved successful. ${ }^{8}$ Methotrexate was compared to cyclophosphamide (non-renal vasculitis alternatively treated with methotrexate trial) and although they were equally effective, there was a delay in remission in the MTX group compared to the cyclophosphamide group, especially in patients with extensive pulmonary involvement with a higher rate of relapse. In the treatment of vasculitis, the difficulty lies more in maintaining the remission rather than inducing it. There are several studies comparing different regimens in maintaining remission. In the WEGENT study, this trial directly compared AZA and MTX in the maintenance of remission after induction with cyclophosphamide. At 29 months, there was no difference in survival between the 2 arms. Rituximab has been approved to treat ANCA-associated vasculitis after the good outcome from different studies that proved its efficacy compared with the standard therapy (RAVE trial). ${ }^{8}$ Rituximab is typically thought to be more effective in the vasculitic phase rather than the granulomatous phase, as recent reports have suggested that rituximab may be less effective for the treatment of necrotizing granulomatous lesions. However, small studies have shown good results with limited GPA. ${ }^{9}$

In conclusion, a limited form of GPA can co-exist with UC, which may confuse the clinical picture, especially if the patient is receiving mesalazine. Awareness of such association may help to minimize this confusion.

\section{References}

1. Mintz R, Feller ER, Bahr RL, Shah SA. Ocular manifestations of inflammatory bowel disease. Inflamm Bowel Dis 2004; 10 : 135-139.

2. Isse N, Nagamatsu Y, Yoshimatsu N, Obata T, Takahara N. Granulomatosis with polyangiitis presenting as an orbital inflammatory pseudo tumor: a case report. JMed case rep 2013; 7: 110 .

3. Kasuga A, Mandai Y, Katsuno T, Sato T, Yamaguchi T, Yokosuka O. Pulmonary complications resembling Wegener's granulomatosis in ulcerative colitis with elevated proteinase-3 anti-neutrophil cytoplasmic antibody. Intern Med 2008; 47: 1211-1214.

4. Fayaz M, Sultan A, Nawaz M, Sultan N. Mesalazine-induced eosinophilic variant of Wegener's granulomatosis in an ulcerative colitis patient. J Ayub Med Coll Abbottabad 2009; 21: 171-173.

5. Miura N, Aoyama R, Kitagawa W, Yamada H, Nishikawa K, Imai H. Proteinase 3-antineutrophil cytoplasmic antibody(PR3-ANCA) positive necrotizing glomerulonephritis after restarting sulphasalazine treatment. Clin Nephrol 2009; 71:74-79.

6. Denissen NH, Peters JG, Masereeuw R, Barrera P.Can sulfasalazine therapy induce or exacerbate Wegener's granulomatosis? Scand J Rheumatol 2008; 37: 72-74.

7. Yano S, Kobayashi K, Kato K, Nishimura K. A limited form of Wegener's granulomatosis with bronchiolitis obliterans organizing pneumonitis-like variant in an ulcerative colitis patient. Intern Med 2002; 41: 1013-1015.

8. Zachary S Wallace, Eli M Miloslevsky. Management of ANCA associated vasculitis. BMJ 2020; 368: 421.

9. Seo P, Specks U, Keogh KA. Efficacy of rituximab in limited Wegener's granulomatosis with refractory granulomatous manifestations. J Rheumatol 2020; 35: 2017-2023. 